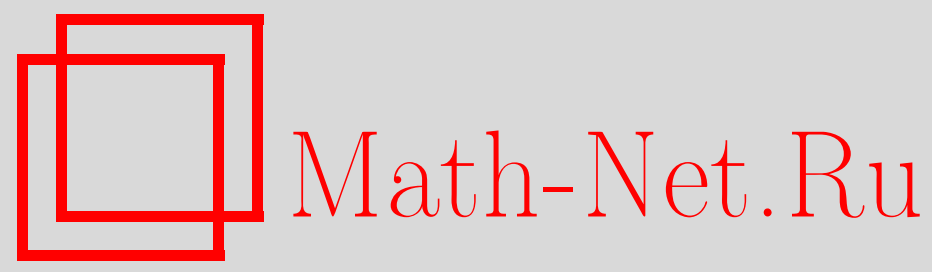

А. Ю. Колесов, А. Н. Куликов, Н. Х. Розов, Аттракторы сингулярно возмущенных параболических систем первой степени негрубости в плоской области, Матем. заметки, 2004, том 75, выпуск 5, 663-669

DOI: https://doi.org/10.4213/mzm62

Использование Общероссийского математического портала Math-Net.Ru подразумевает, что вы прочитали и согласны с пользовательским соглашением http://www . mathnet.ru/rus/agreement

Параметры загрузки:

IP : 18.207 .199 .55

26 апреля 2023 г., 12:53:06

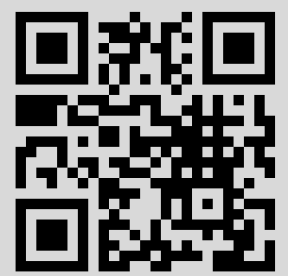




\section{АТТРАКТОРЫ СИНГУЛЯРНО ВОЗМУЩЕННЫХ ПАРАБОЛИЧЕСКИХ СИСТЕМ ПЕРВОЙ СТЕПЕНИ НЕГРУБОСТИ В ПЛОСКОЙ ОБЛАСТИ}

\section{А. Ю. Колесов, А. Н. Куликов, Н. Х. Розов}

Изучается вопрос об аттракторах краевой задачи

$$
u_{t}=\sqrt{\varepsilon}\left(D_{0}+\sqrt{\varepsilon} D_{1}\right) \Delta u+\left(A_{0}+\varepsilon A_{1}\right) u+F(u),\left.\quad u_{x}\right|_{x=0, x=l_{1}}=\left.u_{y}\right|_{y=0, y=l_{2}}=0,
$$

где $0 \leqslant \varepsilon \ll 1, u \in \mathbb{R}^{N}, N \geqslant 3, \Delta$ - оператор Лапласа, - $D_{0}$ - гурвицева матрица. Для такой краевой задачи при определенных предположениях установлено существование любого конечного фиксированного числа устойчивых циклов, если $\varepsilon>0$ выбрано надлежаще малым. Иными словами, показано, что в этой краевой задаче имеет место феномен буферности.

Библиографоял: 8 названий.

1. В области $\Omega=\left\{(x, y): 0 \leqslant x \leqslant l_{1}, 0 \leqslant y \leqslant l_{2}\right\}$ рассмотрим краевую задачу

$$
u_{t}=\sqrt{\varepsilon}\left(D_{0}+\sqrt{\varepsilon} D_{1}\right) \Delta u+\left(A_{0}+\varepsilon A_{1}\right) u+F(u),\left.\quad u_{x}\right|_{x=0, x=l_{1}}=\left.u_{y}\right|_{y=0, y=l_{2}}=0 .
$$

Здесь $\varepsilon>0$ - малый параметр; $u \in \mathbb{R}^{N}, N \geqslant 3$ (при $N=2$ интересующая нас и изучаемая ниже особенность не реализуется); $\Delta$ - оператор Лапласа; матрица $-D_{0}$ гурвищева, а $D_{1}$ - произвольна. Основное предположение, при котором будет изучаться вопрос об аттракторах этой краевой задачи, состоит в следующем: при всех $z>0$ матрица $A_{0}-z D_{0}$ является гурвицевой, причем при малых $z$ она имеет простые собственные значения $\lambda(z), \bar{\lambda}(z): \lambda(0)=i \omega_{0}, \omega_{0}>0$, которые отходят от мнимой оси с касанием первого порядка. Последнее означает, что

$$
\operatorname{Re}\left(D_{0} a, b\right)=0, \quad \varkappa=\operatorname{Im}\left(D_{0} a, b\right) \neq 0, \quad \operatorname{Re}\left(D_{0} a_{1}, b\right)>0,
$$

где $A_{0} a=i \omega_{0} a, A_{0}^{*} b=-i \omega_{0} b,(a, b)=1,(\bar{a}, b)=0,(*, *)$ - евклидово скалярное произведение, а вектор $a_{1}$, удовлетворяющий условию $\left(a_{1}, b\right)=0,-$ решение алгебраического уравнения

$$
A_{0} a_{1}-i \omega_{0} a_{1}=D_{0} a-\left(D_{0} a, b\right) a .
$$

Считаем также, что у матрицы $A_{0}$, кроме пары $\pm i \omega_{0}$, все остальные собственные значения имеют отрицательные действительные части.

Работа выполнена при финансовой поддержке Российского фонда фундаментальных исследований, грант № 02-01-01286. 
Перечислим ограничения, касающиеся вектор-функции $F \in C^{\infty}$. Будем предполагать, что, во-первых, она имеет в нуле тейлоровское разложение вида

$$
F(u)=F_{2}(u, u)+F_{3}(u, u, u)+\cdots,
$$

где $F_{2}, F_{3}, \ldots$ - квадратичная, кубическая и т.д. симметричные формы; во-вторых, $\operatorname{Re} d<0$, где

$$
\begin{aligned}
& d=2\left(F_{2}\left(c_{0}, a\right), b\right)+2\left(F_{2}\left(c_{2}, \bar{a}\right), b\right)+3\left(F_{3}(a, a, \bar{a}), b\right), \\
& c_{0}=-2 A_{0}^{-1} F_{2}(a, \bar{a}), \quad c_{2}=\left[2 i \omega_{0} I-A_{0}\right]^{-1} F_{2}(a, a) .
\end{aligned}
$$

Наконец, сформулируем последнее допущение, характеризующее некоторую общность положения. Это допущение относится к области $\Omega$ : отношение $l_{1}^{2} / l_{2}^{2}$ иррационально.

Вопрос об автоколебаниях параболической краевой задачи, которая получается, если в задаче $(1)$ написать $\varepsilon$ вместо $\sqrt{\varepsilon}$, а матрищу $D_{1}$ отбросить, рассматривался в статьях [1]-[3]. Там изучался "основной случай", когда $\operatorname{Re}\left(D_{0} a, b\right)>0$. Условия же $(2)$ выделяют более тонкую особенность - класс краевых задач "первой степени негрубости", что влечет соответствующую малость диффузии и наличие в краевой задаче (1) матрицы $D_{1}$, характеризующей отход от особенности. Исследование такого типа краевых задач берет свое начало с работы [4], где указанная особенность обнаружена в одной из математических моделей экологии. В работе [4] посредством численного анализа установлено так назьваемое явление буферности, т.е. существование у краевой задачи при подходящем выборе параметров любого конечного числа однотипных аттракторов, и была поставлена проблема теоретического объяснения этого феномена.

В случае краевой задачи для уравнения из (1) “первой степени негрубости” с граничными условиями Неймана на отрезке сформулированная проблема теоретически решена в статье [5]. Точнее говоря, там доказано существование у указанной краевой задачи произвольно фиксированного числа устойчивых гармонических по времени циклов, бифурцирующих при $\varepsilon>0$ из нулевого положения равновесия. В настоящей работе, являющейся продолжением статьи [5], устанавливается, что переход от отрезка к плоской области не приводит к принципиальным изменениям, т.е. явление буферности сохраняется.

Для удобства последующего анализа выполним в (1) замены $x / l_{1} \rightarrow x, y / l_{2} \rightarrow y$. В результате приходим к краевой задаче в квадрате $\Omega_{0}=\{(x, y): 0 \leqslant x \leqslant 1,0 \leqslant y \leqslant 1\}$ :

$$
u_{t}=\sqrt{\varepsilon}\left(D_{0}+\sqrt{\varepsilon} D_{1}\right) L u+\left(A_{0}+\varepsilon A_{1}\right) u+F(u),\left.\quad u_{x}\right|_{x=0, x=1}=\left.u_{y}\right|_{y=0, y=1}=0
$$

где $L=a_{1}^{2} \partial^{2} / \partial x^{2}+a_{2}^{2} \partial^{2} / \partial y^{2}, a_{j}=1 / l_{j}, j=1,2$. При решении проблемы аттракторов получившейся задачи воспользуемся методом квазинормальных форм Ю. С. Колесова [1]-[3]. С этой целью заметим, что при $\varepsilon=0$ и после отбрасывания нелинейности задача (7) допускает семейство периодических решений

$$
u=\eta(x, y) a \exp \left(i \omega_{0} t\right)+\bar{\eta}(x, y) \bar{a} \exp \left(-i \omega_{0} t\right)
$$


где $\eta(x, y)$ - произвольная комплекснозначная функция. Поэтому при $\varepsilon>0$, используя существование семейства (8), ее возможные автоколебательные режимы, бифурцирующие из нулевого состояния равновесия, будем искать в виде ряда по целым степеням $\sqrt{\varepsilon}:$

$$
u=\sqrt{\varepsilon} u_{0}(t, \tau, s, x, y)+\varepsilon u_{1}(t, \tau, s, x, y)+\varepsilon^{3 / 2} u_{2}(t, \tau, s, x, y)+\cdots,
$$

где $\tau=\sqrt{\varepsilon} t, s=\varepsilon t$, все функции периодичны по $t$ с периодом $2 \pi / \omega_{0}$,

$$
u_{0}=\eta_{0}(\tau, s, x, y) a \exp \left(i \omega_{0} t\right)+\bar{\eta}_{0}(\tau, s, x, y) \bar{a} \exp \left(-i \omega_{0} t\right)
$$

а $\eta_{0}$ - пока произвольная (подлежащая определению) комплекснозначная амплитуда. Отметим, что в отличие от случая, рассматривавшегося в [1]-[3], здесь присутствуют два медленных временных масштаба. Причина этого - дополнительное вырождение (2).

Итак, подставим соотношения (9), (10) вместе с тейлоровским разложением (4) в краевую задачу (7) и будем последовательно приравнивать коэффициенты при одинаковых степенях $\varepsilon$. В итоге получим серию линейных неоднородных уравнений вида

$$
\frac{\partial u_{j}}{\partial t}-A_{0} u_{j}=g_{j}(t, \tau, s, x, y), \quad j \geqslant 1,
$$

в которых переменные $\tau, s, x, y$ рассматриваются как параметры.

На первом шаге, т.е. после приравнивания коэффициентов при $\varepsilon$, для $u_{1}$ приходим к уравнению (11) при $j=1$, в котором

$$
g_{1}=-\frac{\partial u_{0}}{\partial \tau}+D_{0} L u_{0}+F_{2}\left(u_{0}, u_{0}\right)
$$

Умножая, далее, скалярно коэффициент при $\exp \left(i \omega_{0} t\right)$ в правой части равенства (12) на вектор $b$ и приравнивая результат к нулю, для фигурирующей в (10) комплекснозначной амплитуды $\eta_{0}$ получаем краевую задачу

$$
\frac{\partial \eta_{0}}{\partial \tau}=i \varkappa L \eta_{0},\left.\quad \frac{\partial \eta_{0}}{\partial x}\right|_{x=0, x=1}=\left.\frac{\partial \eta_{0}}{\partial y}\right|_{y=0, y=1}=0
$$

откуда, в свою очередь, $\eta_{0}$ определяется в виде

$$
\eta_{0}=\sum_{n, k=0}^{\infty} \xi_{n, k}(s) \exp \left(-i \varkappa \omega_{n, k} \tau\right) e_{n, k}(x, y)
$$

Здесь $\omega_{n, k}=\pi^{2}\left(a_{1}^{2} n^{2}+a_{2}^{2} k^{2}\right), e_{n, k}(x, y)=2 \cos (n \pi x) \cos (k \pi y)$, а пока произвольные амплитуды $\xi_{n, k}$ таковы, что сходится ряд с общим членом $\omega_{n, k}^{2}\left|\xi_{n, k}\right|^{2}$ (в этом случае $\eta_{0} \in \mathrm{W}_{2}^{2}$ по переменным $\left.x, y\right)$.

После нахождения $\eta_{0}$ (см. (14)) уравнение (11) при $j=1$ становится разрешимьм в классе периодических по $t$ функций. Несложньй подсчет показьвает, что для $u_{1}$ получается формула

$$
\begin{aligned}
u_{1}= & -L \eta_{0} a_{1} \exp \left(i \omega_{0} t\right)-L \bar{\eta}_{0} \bar{a}_{1} \exp \left(-i \omega_{0} t\right)+\left|\eta_{0}\right|^{2} c_{0}+\eta_{0}^{2} c_{2} \exp \left(2 i \omega_{0} t\right) \\
& +\bar{\eta}_{0}^{2} \bar{c}_{2} \exp \left(-2 i \omega_{0} t\right)+\eta_{1} a \exp \left(i \omega_{0} t\right)+\bar{\eta}_{1} \bar{a} \exp \left(-i \omega_{0} t\right)
\end{aligned}
$$


где $a_{1}$ - решение уравнения (3); $c_{0}, c_{2}$ - векторы $(6) ; \eta_{1}=\eta_{1}(\tau, s, x, y)$ - произвольная комплекснозначная функция.

На втором шаге рассмотрению подлежит уравнение (11) при $j=2$ с неоднородностью

$$
g_{2}=-\frac{\partial u_{0}}{\partial s}-\frac{\partial u_{1}}{\partial \tau}+D_{0} L u_{1}+D_{1} L u_{0}+A_{1} u_{0}+2 F_{2}\left(u_{0}, u_{1}\right)+F_{3}\left(u_{0}, u_{0}, u_{0}\right) .
$$

Из условий его разрешимости в требуемом классе функций с учетом (15), (16) выводим аналогичную (13) краевую задачу

$$
\frac{\partial \eta_{1}}{\partial \tau}=i \varkappa L \eta_{1}+f(\tau, s, x, y),\left.\quad \frac{\partial \eta_{1}}{\partial x}\right|_{x=0, x=1}=\left.\frac{\partial \eta_{1}}{\partial y}\right|_{y=0, y=1}=0
$$

где

$$
f=-\frac{\partial \eta_{0}}{\partial s}+\left(A_{1} a, b\right) \eta_{0}+\left(D_{1} a, b\right) L \eta_{0}-\left(D_{0} a_{1}, b\right) L^{2} \eta_{0}+d\left|\eta_{0}\right|^{2} \eta_{0}
$$

комплексная постоянная $d$ определяется формулой (5), а переменная $s$ рассматривается как параметр.

При анализе краевой задачи (17) существенную роль играет предположение об иррациональности числа $a_{1}^{2} / a_{2}^{2}$, поскольку оно исключает одновременное выполнение равенств вида

$$
\omega_{n, k}=\omega_{n_{1}, k_{1}}+\omega_{n_{2}, k_{2}}-\omega_{n_{3}, k_{3}}, \quad n=n_{1} \pm n_{2} \pm n_{3}, \quad k=k_{1} \pm k_{2} \pm k_{3}
$$

при произвольном выборе комбинаций знаков “+" и “-” во втором и третьем из них (тождественные резонансы, когда $(n, k)=\left(n_{1}, k_{1}\right),\left(n_{2}, k_{2}\right)=\left(n_{3}, k_{3}\right)$, здесь, естественно, не рассматриваются). Таким образом, функция $\eta_{1}$ может быть найдена из (17) в форме формального тригонометрического ряда переменной $\tau$ в том и только в том случае, если в неоднородности (18) отсутствуют гармоники вида $\exp \left(-i \varkappa \omega_{n, k} \tau\right) e_{n, k}(x, y)$, $n, k=0,1, \ldots$. Поэтому приравняем коэффициенты при указанных гармониках к нулю. В итоге для фигурирующих в (14) амплитуд $\xi_{n, k}$ приходим к счетной системе обькновенных дифференциальных уравнений:

$$
\begin{aligned}
\dot{\xi}_{n, k}= & {\left[\gamma_{n, k}+d\left(\frac{9}{4}\left|\xi_{n, k}\right|^{2}+6\left|\xi_{n, 0}\right|^{2}+6\left|\xi_{0, k}\right|^{2}+8\left|\xi_{0,0}\right|^{2}+3 \sum_{\substack{m=1 \\
m \neq k}}^{\infty}\left|\xi_{n, m}\right|^{2}\right.\right.} \\
& +3 \sum_{\substack{m=1 \\
m \neq n}}^{\infty}\left|\xi_{m, k}\right|^{2}+2 \sum_{\substack{m, r=1 \\
m \neq n, r \neq k}}^{\infty}\left|\xi_{m, r}\right|^{2} \\
& \left.\left.+4 \sum_{\substack{r=1 \\
r \neq k}}^{\infty}\left|\xi_{0, r}\right|^{2}+4 \sum_{\substack{m=1 \\
m \neq n}}^{\infty}\left|\xi_{m, 0}\right|^{2}\right)\right] \xi_{n, k}, \quad n, k \geqslant 1, \\
\dot{\xi}_{n, 0}= & {\left[\gamma_{n, 0}+d\left(3\left|\xi_{n, 0}\right|^{2}+8\left|\xi_{0,0}\right|^{2}+4 \sum_{\substack{m=1 \\
m \neq n}}^{\infty}\left|\xi_{m, 0}\right|^{2}+4 \sum_{m=1}^{\infty}\left|\xi_{0, m}\right|^{2}\right.\right.} \\
& \left.\left.+2 \sum_{\substack{m, r=1 \\
m \neq n}}^{\infty}\left|\xi_{m, r}\right|^{2}+3 \sum_{r=1}^{\infty}\left|\xi_{n, r}\right|^{2}\right)\right] \xi_{n, 0}, \quad n \geqslant 1,
\end{aligned}
$$




$$
\begin{aligned}
\dot{\xi}_{0, k}= & {\left[\gamma_{0, k}+d\left(3\left|\xi_{0, k}\right|^{2}+8\left|\xi_{0,0}\right|^{2}+4 \sum_{\substack{m=1 \\
m \neq k}}^{\infty}\left|\xi_{0, m}\right|^{2}+4 \sum_{m=1}^{\infty}\left|\xi_{m, 0}\right|^{2}\right.\right.} \\
& \left.\left.+2 \sum_{\substack{m, r=1 \\
r \neq k}}^{\infty}\left|\xi_{m, r}\right|^{2}+3 \sum_{m=1}^{\infty}\left|\xi_{m, k}\right|^{2}\right)\right] \xi_{0, k}, \quad k \geqslant 1, \\
\dot{\xi}_{0,0}= & {\left[\gamma_{0,0}+d\left(4\left|\xi_{0,0}\right|^{2}+4 \sum_{m=1}^{\infty}\left|\xi_{0, m}\right|^{2}+4 \sum_{m=1}^{\infty}\left|\xi_{m, 0}\right|^{2}+2 \sum_{m, r=1}^{\infty}\left|\xi_{m, r}\right|^{2}\right)\right] \xi_{0,0}, }
\end{aligned}
$$

где $\gamma_{n, k}=\left(A_{1} a, b\right)-\omega_{n, k}\left(D_{1} a, b\right)-\omega_{n, k}^{2}\left(D_{0} a_{1}, b\right)$, а точка - дифференцирование по $s$.

Получившаяся система позволяет разобраться с проблемой существования и устойчивости у исходной задачи (7) циклов и торов, бифурцирующих из нулевого состояния равновесия. Для того чтобы сформулировать строгий результат, будем считать, что в качестве фазового пространства задачи $(7)$ взято $\stackrel{\circ}{\mathrm{W}} \underset{2}{2}\left(\Omega_{0} ; \mathbb{R}^{N}\right)$, где $\stackrel{\circ}{\mathrm{W}}{ }_{2}^{2}-$ замькание в метрике $\mathrm{W}_{2}^{2}$ линеала гладких вектор-функций, удовлетворяющих граничным условиям из (7). Что же касается системы (19)-(22), то в силу условия $\operatorname{Re}\left(D_{0} a_{1}, b\right)>0$ (см. (2)) она представляет собой абстрактную параболическую систему [6] в пространстве последовательностей $\xi=\left\{\xi_{n, k}, \bar{\xi}_{n, k}\right\}$, для которых конечна норма

$$
\|\xi\|=\left(\left|\xi_{0,0}\right|^{2}+\sum_{n, k: n+k>0} \omega_{n, k}^{2}\left|\xi_{n, k}\right|^{2}\right)^{1 / 2} .
$$

Предположим, далее, что система (19)-(22) имеет автомодельный тор вида

$$
\xi_{n_{j}, k_{j}}(s)=\rho_{j} \exp \left(i \psi_{j} s\right), \quad j=1, \ldots, p, \quad \xi_{n, k}=0 \quad \text { при }(n, k) \neq\left(n_{j}, k_{j}\right),
$$

где $p \geqslant 1, \rho_{j}>0, \psi_{j} \in \mathbb{R}$. Справедлива следующая

ТЕОрема. Любому автомодельному тору системы (19)-(22) вида (23), әкспоненииально орбитально устойчивому или дихотомичному, в краевой задаче (7) при всех достаточно малых $\varepsilon>0$ соответствует $p$-мерный инвариантный тор той же устойчивости, задающийся равенствами

$$
\begin{gathered}
u=\sqrt{\varepsilon} \sum_{j=1}^{p}\left[a \exp \left(i \varphi_{j}\right)+\bar{a} \exp \left(-i \varphi_{j}\right)\right] \rho_{j} e_{n_{j}, k_{j}}(x, y)+\varepsilon u_{*}(\varphi, x, y, \varepsilon), \\
\frac{d \varphi_{j}}{d t}=\omega_{0}-\sqrt{\varepsilon} \varkappa \omega_{n_{j}, k_{j}}+\varepsilon \psi_{j}+\varepsilon^{3 / 2} \psi_{*, j}(\varphi, \varepsilon), \quad j=1, \ldots, p .
\end{gathered}
$$

Здесь $\varphi=\operatorname{colon}\left(\varphi_{1}, \ldots, \varphi_{p}\right)$, a $2 \pi$-периодические по $\varphi$ функиии $u_{*} u \psi_{*}=\operatorname{colon}\left(\psi_{*, 1}\right.$, $\ldots, \psi_{*, p}$ ) ограничены равномерно по $\varepsilon, \varphi$ (вместе слюбым фиксированным числом производных по $\varphi)$ в метрике $\stackrel{\circ}{\mathrm{W}} \underset{2}{2}\left(\Omega_{0} ; \mathbb{R}^{N}\right)$ и $\mathbb{R}^{p}$ соответственно.

Подробно обоснование сформулированной теоремы мы здесь воспроизводить не будем, поскольку оно без каких-либо дополнительных трудностей реализуется по описанной в [5] схеме с привлечением развитых в [3] методов доказательства существования инвариантных торов в сингулярно возмущенных системах. 
2. Итак, вопрос об аттракторах краевой задачи (7) сводится к аналогичному вопросу для ее квазинормальной формы (19)-(22). За аттракторы же последней отвечает, в свою очередь, конечномерная система, получающаяся из (19)-(22), если положить равными нулю те компоненты $\xi_{n, k}$, для которых $\tau_{n, k}^{\prime}=\operatorname{Re} \gamma_{n, k} \leqslant 0$. Действительно, из структуры уравнений (19)-(22) и условия $\operatorname{Re} d<0$ следует, что модули упомянутых компонент стремятся к нулю при $s \rightarrow \infty$.

Рассмотрим сначала две простейшие ситуации. Предположим сначала, что при некоторых фиксированных $n, k \geqslant 0$ выполняется условие самовозбуждения $\tau_{n, k}^{\prime}>0$. Тогда система (19)-(22) имеет, очевидно, автомодельньй цикл

$$
\xi_{n, k}(s)=\rho_{n, k} \exp \left(i \psi_{n, k} s\right), \quad \xi_{m, r}(s) \equiv 0 \quad \text { при } \quad(m, r) \neq(n, k)
$$

где $\rho_{n, k}=\sqrt{-4 \tau_{n, k}^{\prime} /(9 \operatorname{Re} d)}, \psi_{n, k}=\operatorname{Im} \gamma_{n, k}+\frac{9}{4} \rho_{n, k}^{2} \operatorname{Im} d$. Нетрудно вьписать также и условия устойчивости данного цикла. Не останавливаясь на соответствующих вычислениях, заметим, что эти условия заведомо справедливы, если возбуждена только мода $e_{n, k}(x, y)$, т.е. $\tau_{m, r}^{\prime}<0$ при $(m, r) \neq(n, k)$.

Предположим теперь, что, к примеру, $\tau_{1,2}^{\prime}>0, \tau_{2,1}^{\prime}>0$, а все остальные моды не возбуждены. Тогда за аттракторы квазинормальной формы отвечает система

$$
\begin{aligned}
& \dot{\xi}_{1,2}=\left[\gamma_{1,2}+d\left(\frac{9}{4}\left|\xi_{1,2}\right|^{2}+2\left|\xi_{2,1}\right|^{2}\right)\right] \xi_{1,2}, \\
& \dot{\xi}_{2,1}=\left[\gamma_{2,1}+d\left(2\left|\xi_{1,2}\right|^{2}+\frac{9}{4}\left|\xi_{2,1}\right|^{2}\right)\right] \xi_{2,1},
\end{aligned}
$$

которая при подходящем выборе параметров может иметь устойчивьй двумерньй автомодельньй тор. А отсюда и из сформулированной вьше теоремы следует, что устойчивый двумерный тор вида (24) имеет в этом случае и исходная задача (7).

В заключение покажем, что при условиях

$$
\operatorname{Re}\left(A_{1} a, b\right)>0, \quad D_{0}=\nu D_{0,0}, \quad D_{1}=\nu D_{1,0}, \quad \operatorname{Re}\left(D_{1,0} a, b\right) \geqslant 0, \quad \nu>0,
$$

и при уменьшении параметра $\nu$ в рамках краевой задачи (7) реализуется феномен буферности. С этой целью рассмотрим сразу предельный случай $\nu=0$, т.е. в системе (19)-(22) положим $\gamma_{n, k}=\left(A_{1} a, b\right), n, k \geqslant 0$. Нетрудно заметить, что при выполнении первого из условий (26) получившаяся система имеет счетное число устойчивых автомодельных циклов $(25)$ с номерами $(n, 0), n \geqslant 1 ;(0, k), k \geqslant 1$ и $(0,0)$. Это означает, что при условиях (26) и при подходящем уменьшении параметров $\varepsilon$ и $\nu$ исходная краевая задача (7) имеет любое наперед заданное конечное число устойчивых циклов, зависящих только от одной пространственной переменной.

Полученный результат на первьй взгляд весьма парадоксален: при пропорциональном уменьшении коэффициентов диффузии, т.е. при углублении степени сингулярности, происходит, казалось бы, упрощение динамики. Но это упрощение носит временньй характер, так как уже при

$$
\nu=\nu_{0} \sqrt{\varepsilon}, \quad \nu_{0}=\text { const }>0
$$


и при условиях (26) описанный вьше метод квазинормализации приводит к краевой задаче

$$
\frac{\partial \eta_{0}}{\partial s}=i \nu_{0} \varkappa_{0} L \eta_{0}+\left(A_{1} a, b\right) \eta_{0}+d\left|\eta_{0}\right|^{2} \eta_{0},\left.\quad \frac{\partial \eta_{0}}{\partial x}\right|_{x=0, x=1}=\left.\frac{\partial \eta_{0}}{\partial y}\right|_{y=0, y=1}=0
$$

где $\varkappa_{0}=\operatorname{Im}\left(D_{0,0} a, b\right)$, динамика которой достаточно сложна.

Действительно, в данном случае, во-первых, сохраняется стандартное утверждение о соответствии (см. [1]-[3]): каждому экспоненциально орбитально устойчивому циклу

$$
\eta_{0}=\eta_{0}^{*}(x, y) \exp \left(i \alpha_{0} s\right), \quad \alpha_{0}=\text { const } \in \mathbb{R},
$$

краевой задачи (28) в исходной задаче (7) при всех достаточно малых $\varepsilon>0$ и при условиях $(26),(27)$ отвечает устойчивьй цикл с асимптотикой $(9),(10),(29)$; во-вторых, сама квазинормальная форма (28) имеет счетные наборы циклов вида (29), зависящих только от одной пространственной переменной $x$ или $y$, причем при $\nu_{0} \gg 1$ можно гарантировать устойчивость любого конечного их числа (см. [7]). В случае же $\nu_{0} \rightarrow 0$ указанные циклы, вообще говоря, теряют устойчивость и происходит усложнение динамики (что косвенным образом следует из проделанного в [8] численного анализа).

\section{СПИСОК ЦИТИРОВАННОЙ ЛИТЕРАТУРЫ}

[1] Колесов Ю. С. Динамика биологических популяций // Межвуз. сб.: Горьковский ун-т, 1984. C. $28-34$.

[2] Колесов Ю. С. Об одной бифуркационной теореме в теории автоколебаний распределенных систем // Дифференц. уравнения. 1985. Т. 21. №10. С. 1709-1713.

[3] Колесов Ю. С. Бифуркация инвариантных торов параболических систем с малой дифффузией // Матем. сб. 1993. Т. 184. № 3. С. 121-136.

[4] Захаров А. А., Колесов Ю. С. // Нелинейные колебания и экология. Ярославл: ЯрГУ, 1984. C. 3-15.

[5] Колесов А. Ю. Бифуркация периодических решений сингулярно возмущенных параболических систем первой степени негрубости // Укр. матем. журн. 1990. Т. 42. № 8. С. 1037-1042.

[6] Соболевский П. Е. Об уравнениях параболического типа в банаховом пространстве // Tp. MMO. 1961. T. 10. C. 297-350.

[7] Колесов А. Ю., Розов Н. Х. Особенности динамики уравнения Гинзбурга-Ландау в плоской области // ТМФ. 2000. Т. 125. №2. С. 205-220.

[8] Колесов Ю. С., Харьков А. Е. Аттракторы сингулярно возмущенных систем типа реакция-диффузия с крайней точкой поворота в плоской области // Докл. РАН. 2001. Т. 377. № 2. C. $173-175$.

(А. Ю. Колесов, А. Н. Куликов) Ярославский государственный

Поступило университет им. П. Г. Демидова 25.03.2002 (Н. Х. Розов) Московский государственный университет им. М. В. Ломоносова 